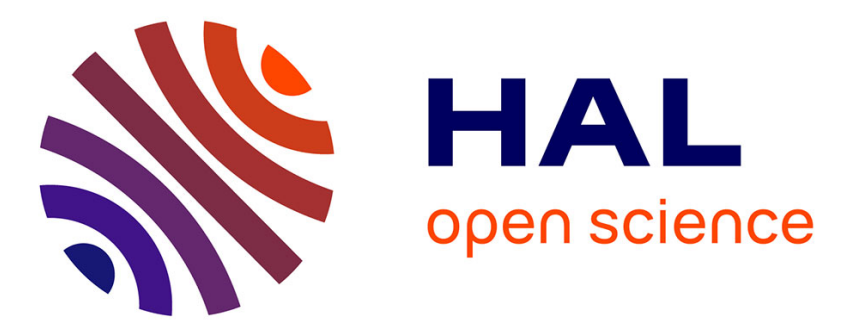

\title{
Contrôle de l'élaboration des thyristors par thermocapacitance: caractérisation de centres recombinants induits
}

J. Barbolla, L. Bailon, J.C. Brabant, M. Pugnet, M. Brousseau

\section{- To cite this version:}

J. Barbolla, L. Bailon, J.C. Brabant, M. Pugnet, M. Brousseau. Contrôle de l'élaboration des thyristors par thermocapacitance: caractérisation de centres recombinants induits. Revue de Physique Appliquée, 1976, 11 (3), pp.403-408. 10.1051/rphysap:01976001103040300 . jpa-00244075

\section{HAL Id: jpa-00244075 https://hal.science/jpa-00244075}

Submitted on 1 Jan 1976

HAL is a multi-disciplinary open access archive for the deposit and dissemination of scientific research documents, whether they are published or not. The documents may come from teaching and research institutions in France or abroad, or from public or private research centers.
L'archive ouverte pluridisciplinaire HAL, est destinée au dépôt et à la diffusion de documents scientifiques de niveau recherche, publiés ou non, émanant des établissements d'enseignement et de recherche français ou étrangers, des laboratoires publics ou privés. 


\title{
CONTRÔLE DE L'ÉLABORATION DES THYRISTORS PAR THERMOCAPACITANCE : CARACTÉRISATION DE CENTRES RECOMBINANTS INDUITS
}

\author{
J. BARBolla, L. BAILON, J. C. BRABANT, M. PUGNET et M. BROUSSEAU
}

Laboratoire de Physique des Solides (*), I. N. S. A., Département de Physique, Avenue de Rangueil, 31077 Toulouse-Cedex, France

(Reçu le 12 décembre 1975, accepté le 23 janvier 1976)

\begin{abstract}
Résumé. - Nous appliquons la thermocapacitance et la méthode des transitoires de capacité dans l'obscurité aux dispositifs de puissance au silicium. Nous utilisons ces méthodes pour caractériser les niveaux d'énergie profonds induits à chaque étape de la fabrication de thyristors. Nous trouvons trois niveaux à $E_{\mathrm{C}}-(160 \pm 5) \mathrm{meV}, E_{\mathrm{C}}-(257 \pm 4) \mathrm{meV}$ et $E_{\mathrm{c}}-(542 \pm 4) \mathrm{meV}$. Le second et le troisième niveau sont associés à des centres recombinants induits par la trempe (Quenched-in centers).

Abstract. - We adapt the thermally stimulated capacitance and dark transient capacitance technique to the study of silicon power devices. We use this method for the characterization of deep levels which appear at each stage of thyristor fabrication. We find three levels at $E_{\mathrm{C}}-(160 \pm 5) \mathrm{meV}$, $E_{\mathrm{c}}-(257 \pm 4) \mathrm{meV}$ and $E_{\mathrm{c}}-(542 \pm 4) \mathrm{meV}$. The second and third levels are associated with quench induced recombination centers (Quenched-in centers).
\end{abstract}

1. Introduction. - La caractérisation des centres recombinants dans les dispositifs de puissance au silicium revêt aujourd'hui une grande importance pour l'industrie électronique. En effet, la durée de vie en direct dans la zone faiblement dopée des dispositifs est couramment réglée par l'introduction d'impuretés métalliques par diffusion : les états électroniques localisés associés à ces impuretés jouent un rôle essentiel dans les phénomènes de génération-recombinaison. Le bon compromis courte durée de vie en direct-faible courant de génération en inverse peut être réalisé par le choix d'impuretés métalliques adéquates [1]. Par contre la présence d'impuretés incontrôlées, même à l'état de traces $\left(10^{12} \mathrm{~cm}^{-3}\right.$ par exemple), et introduisant un niveau d'énergie au voisinage du milieu de la bande interdite, peut être à l'origine d'un courant de génération inverse élevé [1].

Les méthodes classiques de caractérisation électrique (effet Hall, photoconductivité, résistivité en fonction de la température) ne peuvent pas être appliquées sur des dispositifs finis.

Une technique de caractérisation fondée sur le principe de la stimulation thermique de la capacité d'une jonction [2] a été adaptée à l'analyse des centres recombinants dans les dispositifs de puissance au silicium. Cette technique permet :

- La mesure précise (incertitude de quelques meV)

(*) Associé au C. N. R. S. de la position des niveaux d'énergie dans la bande interdite ;

- La mesure du profil de dopage en centres recombinants ;

- La.caractérisation d'impuretés incontrôlées à des densités très faibles $\left(\gtrsim 10^{12} \mathrm{~cm}^{-3}\right)$.

Le premier objectif de ce travail est de définir les conditions de son application aux thyristors, à diverses étapes de leur fabrication : ceci est fait au paragraphe 2 .

Le second objectif est de caractériser les centres recombinants pouvant apparaître à ces diverses étapes : impuretés résiduelles, complexes formés au cours d'une étape à haute température ( $\left.1200^{\circ} \mathrm{C}\right)$, centres induits par les traitements thermiques [3], [4]. Nos résultats expérimentaux sont exposés au paragraphe 4 et comparés à ceux de Yau et Sah [3] qui ont travaillé sur des jonctions $\mathrm{p}^{+} \mathrm{n}$. Nos résultats sont relatifs à la caractérisation bien précise d'une technologie. A ce titre, ils illustrent l'intérêt de la technique employée.

2. La technique expérimentale. - 2.1 THERMOCAPACITANCE [ET |TRANSITOIRES DE |CAPACITÉ SUR JONCTION PN. - 2.1.1 Introduction. - Les techniques de mesure des coefficients d'émission d'électrons et de trous par les centres profonds ont été discutées en détail.par C. T. Sah [5]. Nous rappelons ici le principe de la thermocapacitance [2] et de la méthode des transitoires de capacité dans l'obscurité. Nous raisonnons dans l'hypothèse où un seul centre profond est présent. 
Dans le cas contraire, les critères de validité établis restent valables pour chaque centre.

La capacité $h$. f. d'une jonction $p-n$ abrupte $\left(p^{+} n\right.$ ou $\mathrm{n}^{+} \mathrm{p}$ ) polarisée en inverse et mesurée avec un signal de faible amplitude par rapport à la polarisation est donnée par :

$$
C=\frac{\varepsilon A}{W}
$$

ce qui, pour une distribution uniforme de charges dans la zone déserte, peut être écrit :

$$
C=\left[\frac{\varepsilon A^{2}\left(e N_{\mathrm{D}}+q_{\mathrm{i}} N_{\mathrm{i}}\right)}{2\left(V_{\mathrm{B}}+V\right)}\right]^{1 / 2}
$$

où $q_{\mathrm{i}}$ et $N_{\mathrm{i}}$ sont respectivement la charge algébrique et la densité du centre ionisé d'indice i; $N_{\mathrm{D}}$ est la densité de donneurs peu profonds ; $e$ est la valeur absolue de la charge de l'électron ; $\varepsilon$ est la constante diélectrique du silicium ; $A$ est la surface de la jonction; $W$ est la largeur de la zone déserte ; $V$ est la polarisation inverse appliquée ; $V_{\mathbf{B}}$ est la hauteur de la barrière de potentiel lorsque $V=0$.

La fréquence du signal de mesure doit être suffisamment élevée pour que la charge des centres profonds dans la zone déserte ne réponde pas à l'excitation [6] :

$$
\omega \gg \frac{1}{\tau_{T}}
$$

$\tau_{T}$ est le plus petit des temps de charge et de décharge des centres profonds dans la zone déserte. Le temps de charge est associé à la mise brusque des centres déchargés dans l'environnement du gaz de porteurs libres de la base. Le temps de décharge est associé à un retrait brusque de ce gaz.

2.1.2 La thermocapacitance. - La thermocapacitance est mise en œuvre de la façon suivante :

1. On abaisse la température de la diode non polarisée jusqu'à ce que les coefficients d'émission des porteurs par les centres profonds soient négligeables (très couramment $T=77 \mathrm{~K}$ ).

2. On polarise la diode en inverse, de sorte que dans la zone déserte les centres profonds soient peuplés d'électrons (pour une diode $\mathrm{p}^{+} \mathrm{n}$ ) ou de trous (pour une diode $\mathrm{n}^{+} \mathrm{p}$ ) (voir Fig. 1).

3. En maintenant la polarisation inverse constante, on chauffe l'échantillon de sorte que sa température s'accroisse linéairement avec le temps. La capacité $h$. f. est enregistrée ; elle subit de brusques accroissements (avec point d'inflexion) chaque fois que l'énergie thermique provoque l'émission de porteurs libres à partir d'un niveau d'énergie associé à un état électronique localisé (voir Fig. 1 et Fig. 2).

La température $T_{\mathrm{i}}$ correspondant à un point d'inflexion est dépendante de l'énergie de ce niveau et de la vitesse de chauffage (Fig. 2).

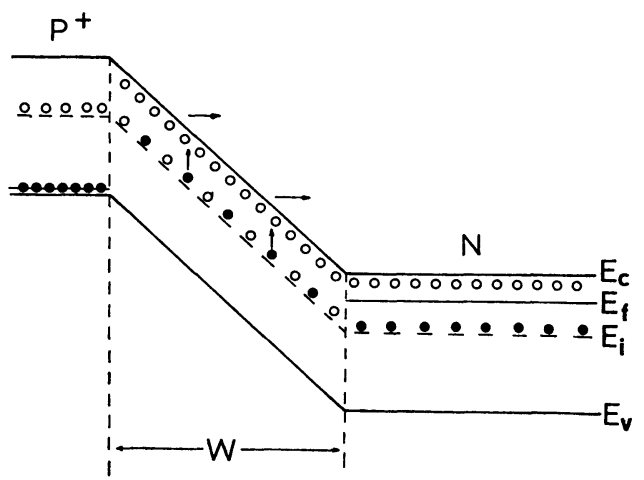

Fig. 1. - Schéma de bandes d'une jonction $\mathrm{p}^{+} \mathrm{n}$ polarisée en inverse : $E_{\mathrm{V}}$ : bord supérieur de la bande de valence ; $E_{\mathrm{C}}:$ bord inférieur de la bande de conduction ; $E_{\mathrm{F}}$ : niveau de Fermi dans la zone $\mathrm{N} ; E_{1}$ : niveau d'énergie profond.

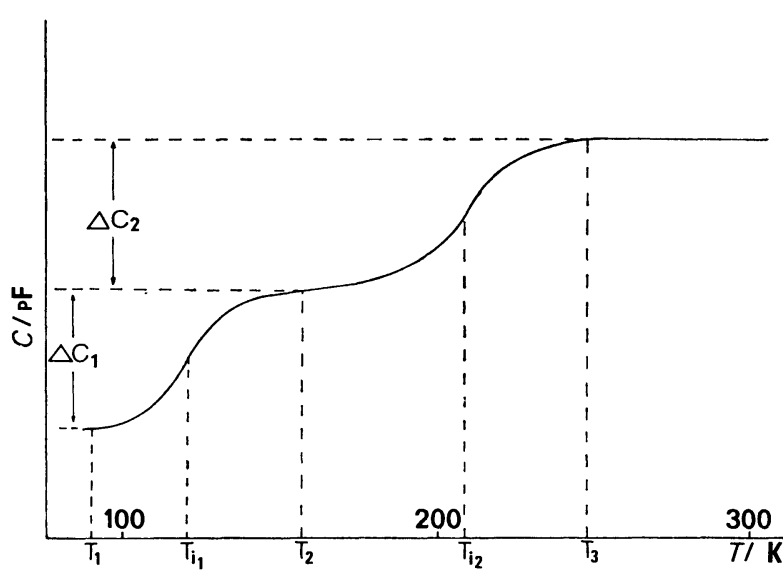

Fig. 2. - Courbe théorique capacité-température pour le cas où deux niveaux apparaissent. $T_{i_{1}}$ et $T_{i_{2}}$ sont les températures respectives correspondant aux points d'inflexion de la capacité pour les niveaux 1 et 2 .

Afin que la technique de capacité thermostimulée soit applicable simplement, il est souhaitable que les variations $\Delta C_{1}$ et $\Delta C_{2}$ restent faibles devant la capacité totale [5]. Cela suppose :

$$
N_{\mathrm{i}} \ll N_{\mathrm{D}} .
$$

Dans ces conditions :

$$
\frac{\Delta C_{\mathrm{i}}}{C} \simeq \frac{1}{2} \frac{N_{\mathrm{i}}}{N_{\mathrm{D}}} .
$$

2.1.3 Mesure des profils de dopage. - La connaissance des profils de dopage pour le centre profond associé à un niveau peut être déduite de la formule :

$$
\frac{\mathrm{d}\left(1 / C^{2}\right)}{\mathrm{d} V}=\frac{2}{\varepsilon A^{2}\left[e N_{\mathrm{D}}+q_{\mathrm{i}} N_{\mathrm{i}}(W)\right]}
$$

applicable pour une jonction abrupte, et des profils $N_{\mathrm{i}}(x)$ quelconques.

En effet, si pour le niveau i on relève les courbes $C(V)$ pour deux températures $T_{1}$ et $T_{2}$ telles que

$$
T_{1}<T_{\mathrm{i}}<T_{2}
$$


(voir Fig. 2 pour le cas du niveau 1), on a :

$$
N_{\mathrm{i}}(W)=\frac{2}{e \varepsilon A^{2}}\left[\frac{\mathrm{d} V}{\mathrm{~d}\left(1 / C_{2}^{2}\right)}-\frac{\mathrm{d} V}{\mathrm{~d}\left(1 / C_{1}^{2}\right)}\right]
$$

$C_{1}(V)$ et $C_{2}(V)$ sont les capacités h. f. aux températures $T_{1}$ et $T_{2}$ respectivement.

$N_{\mathrm{i}}(W)$ est la densité du centre profond associé au niveau i à l'abscisse $W$ mesurée par rapport à la jonction métallurgique.

2.1.4 Mesure des coefficients d'émission. - Les coefficients d'émission et de capture sont reliés, d'après le principe du bilan détaillé, par :

$$
e_{\mathrm{n}}^{\mathrm{t}}=C_{\mathrm{n}}^{\mathrm{t}} \cdot N_{\mathrm{C}} \cdot \exp \left(E_{\mathrm{i}}-E_{\mathrm{C}}\right) / k T \quad \text { (voir réf. }
$$

où $e_{\mathrm{n}}^{\mathrm{t}}$ et $C_{\mathrm{n}}^{\mathrm{t}}=\sigma_{\mathrm{n}} v_{\mathrm{th}}$ sont les coefficients d'émission et de capture thermiques d'électrons par les centres profonds ;

$\sigma_{\mathrm{n}}\left(\mathrm{cm}^{2}\right)$ est la section de capture, dont la dépendance en température peut varier de $T^{-4}$ à $T^{0}$; le cas d'une dépendance en $T^{0}$ correspond à la capture par des centres neutres;

$v_{\text {th }}$ est la vitesse thermique moyenne proportionnelle à $T^{1 / 2}$;

$E_{\mathrm{i}}$ est l'énergie du niveau profond;

$E_{\mathrm{C}}$ est l'énergie du bas de bande de conduction;

$N_{\mathrm{C}}$ est la densité équivalente d'états dans la bande de conduction, proportionnelle à $T^{3 / 2}$.

Dans ces conditions et pour l'émission d'électrons à partir de centres chargés $(-e)$, et qui deviennent neutres dans l'émission, on peut écrire :

$$
e_{\mathrm{n}}^{\mathrm{t}}=A\left(\frac{T}{300}\right)^{2} \exp -\frac{E_{\mathrm{C}}-E_{\mathrm{i}}}{k T}
$$

avec $A$ indépendant de la température.

La mesure des coefficients d'émission se fait par la technique des transitoires de capacité dans l'obscurité [5] qui est mise en œuvre de la façon suivante : Pour diverses températures fixées, voisines de $T_{\mathrm{i}}$ on enregistre les transitoires de capacité produits par le passage brusque à l'instant $t=0$, d'une polarisation nulle à une polarisation inverse donnée. Les températures sont choisies en sorte que ces transitoires isothermes soient aisément enregistrés, avec la plus grande précision, tout en recouvrant le maximum d'ordres de grandeur.

La relation coefficient d'émission des porteurscapacité, pour une jonction $\mathrm{p}^{+} \mathrm{n}$, dans le cas d'un niveau donneur, est [3] :

$C^{2}(t)=\frac{e \varepsilon A^{2}}{2\left(V_{\mathrm{B}}+V\right)}\left[N_{\mathrm{D}}+N_{\mathrm{i}} \frac{e_{\mathrm{n}}^{\mathrm{t}}}{e_{\mathrm{n}}^{\mathrm{t}}+\frac{e_{\mathrm{p}}^{\mathrm{t}}}{\mathrm{t}}}\left(1-\mathrm{e}^{-t / \tau}\right)\right]$

avec

$$
\tau=\frac{1}{e_{\mathbf{n}}^{\mathfrak{t}}+e_{\mathrm{p}}^{\mathfrak{t}}}
$$

où $e_{\mathrm{n}}^{\mathrm{t}}$ et $e_{\mathrm{p}}^{\mathrm{t}}$ sont les coefficients d'émission thermique d'électrons et de trous.

De même, pour une jonction $\mathrm{p}^{+} \mathrm{n}$ dans le cas d'un niveau accepteur:

$$
\begin{aligned}
C^{2}(t)=\frac{e \varepsilon A^{2}}{2\left(V_{\mathrm{B}}+V\right)}\left[N_{\mathrm{D}}-\right. & N_{\mathrm{i}} \frac{e_{\mathrm{p}}^{\mathrm{t}}}{e_{\mathrm{n}}^{\mathrm{t}}+e_{\mathrm{p}}^{\mathrm{t}}}- \\
& \left.-N_{\mathrm{i}} \frac{e_{\mathrm{n}}^{\mathrm{t}}}{e_{\mathrm{n}}^{\mathrm{t}}+e_{\mathrm{p}}^{\mathrm{t}}} \mathrm{e}^{-t / \tau}\right] .
\end{aligned}
$$

Ces équations supposent que les densités $N_{\mathrm{D}}$ et $N_{\mathrm{i}}$ sont constantes dans la zone $n$. Dans le cas contraire, Sah et $\mathrm{Fu}$ [7] ont montré que la méthode qui consiste à extraire $\left(e_{\mathrm{n}}^{\mathrm{t}}+e_{\mathrm{p}}^{\mathrm{t}}\right)^{-1}$ de la courbe $C(t)$ reste valable pourvu que $N_{\mathrm{i}} \ll N_{\mathrm{D}}$. En effet, cette inégalité garantit le caractère exponentiel des transitoires. Avec cette condition, pour un niveau accepteur ou donneur, nous avons :

$$
\Delta C(t)=C(t)-C(\infty) \simeq-\Delta C_{0} \mathrm{e}^{-t / \tau}
$$

où

$$
\Delta C_{0}=\frac{A}{2}\left(\frac{e \varepsilon N_{\mathrm{D}}}{2\left(V_{\mathrm{B}}+V\right)}\right)^{1 / 2} \frac{e_{\mathrm{n}}^{\mathrm{t}}}{e_{\mathrm{n}}^{\mathrm{t}}+e_{\mathrm{p}}^{\mathrm{t}}} \frac{N_{\mathrm{i}}}{N_{\mathrm{D}}} .
$$

Si l'inégalité $N_{\mathrm{i}} \ll N_{\mathrm{D}}$ n'est pas réalisée, mais seulement $N_{\mathrm{i}}<N_{\mathrm{D}}$ (par exemple $N_{\mathrm{i}} \sim \frac{1}{3} N_{\mathrm{D}}$ ) la courbe $C(t)$ n'est plus exponentielle car la variation $\Delta C_{i}$ n'est plus proportionnelle à $N_{\mathrm{i}}(3)$. Cependant $\left(e_{\mathrm{n}}^{\mathrm{t}}+e_{\mathrm{p}}^{\mathrm{t}}\right)^{-1}$ peut être extrait de la fin du transitoire $C(t)$. En effet, la fin du transitoire est exponentielle à partir de l'instant où la densité $n_{\mathrm{i}}(t)$ d'électrons occupant le niveau d'énergie $E_{\mathrm{i}}$ est telle que $n_{\mathrm{i}}(t) \ll N_{\mathrm{D}}$.

De plus, dans le cas de l'émission d'électrons nous avons

$$
e_{\mathrm{n}}^{\mathrm{t}} \gg e_{\mathrm{p}}^{\mathrm{t}} \quad \text { d'où } \quad \tau=\frac{1}{e_{\mathrm{n}}} .
$$

2.2 THERMOCAPACITANCE ET TRANSITOIRES DE CAPACITÉ SUR DES DISPOSITIFS DE PUISSANCE. - La première condition pour l'application de la thermocapacitance est évidemment que la zone faiblement dopée du dispositif contienne assez de porteurs libres pour qu'il puisse y avoir modulation des largeurs de charge d'espace, c'est-à-dire :

$$
\omega \tau_{\mathrm{D}} \ll 1
$$

$\tau_{\mathrm{D}}$ étant le temps de relaxation diélectrique du gaz de porteurs dans la zone faiblement dopée. Cette condition est en pratique largement réalisée, pour les dopages $10^{13}-10^{14} \mathrm{~cm}^{-3}$ et la fréquence $1 \mathrm{MHz}$, lorsque $T>T_{\mathrm{e}} . T_{\mathrm{e}}$ est la température d'ionisation des donneurs peu profonds de la zone faiblement dopée. La thermocapacitance n'est donc applicable que pour $T>T_{\mathrm{e}}$.

L'étude des thyristors aux diverses étapes de leur fabrication s'appuie sur l'analyse de la capacité des structures $\mathrm{p}^{+} \mathrm{np}^{+}$et $\mathrm{p}^{+} \mathrm{np}^{+} \mathrm{n}^{++}$successivement. 
Contribution de la jonction $\mathrm{p}^{+} \mathrm{n}^{++}$à la capacité totale du dispositif $\mathrm{p}^{+} \mathrm{np}^{+} \mathrm{n}^{++}$. Les dopages que nous utilisons pour la jonction $\mathrm{p}^{+} \mathrm{n}^{++}$(voir Fig. 5) sont toujours beaucoup plus élevés que ceux des jonctions $\mathrm{p}^{+} \mathrm{n}$ et $\mathrm{np}^{+}$. Il en résulte, quelle que soit la polarisation :

- Une épaisseur $W$ de la zone de charge d'espace très faible pour cette jonction, d'où une capacité $\varepsilon A / W$ très grande ;

- Une conductivité élevée, susceptible de shunter cette capacité.

Dans ces conditions, la contribution lde la jonction $\mathrm{p}^{+} \mathrm{n}^{++}$est toujours négligeable et le problème se ramène à la mesure de la capacité d'une structure $\mathrm{p}^{+} \mathrm{np}{ }^{+}$.

Contribution de la jonction $\mathrm{np}^{+}$à la capacité d'une structure $\mathrm{p}^{+} \mathrm{np}^{+}$polarisée avec le $(+)$à droite. Soit $C_{1}$ et $C_{2}$ les capacités respectives des jonctions $\mathrm{p}^{+} \mathrm{n}$ et $\mathrm{np}^{+}$. A basse température, les deux capacités sont en série et la capacité de la structure $\mathrm{p}^{+} \mathrm{np}^{+}$est donc :

$$
C=\frac{C_{1} C_{2}}{C_{1}+C_{2}}
$$

Lorsque la température croît, le courant de fuite du dispositif augmente et la capacité $C_{2}$ est progressivement shuntée. La jonction $\mathrm{np}^{+}$se manifeste donc par une transition de la capacité totale de la valeur $\frac{C_{1} C_{2}}{C_{1}+C_{2}}$ à la valeur $C_{1}$. Sur la figure 3 , cet accroissement de capacité s'effectue entre les températures $\theta_{1}$ et

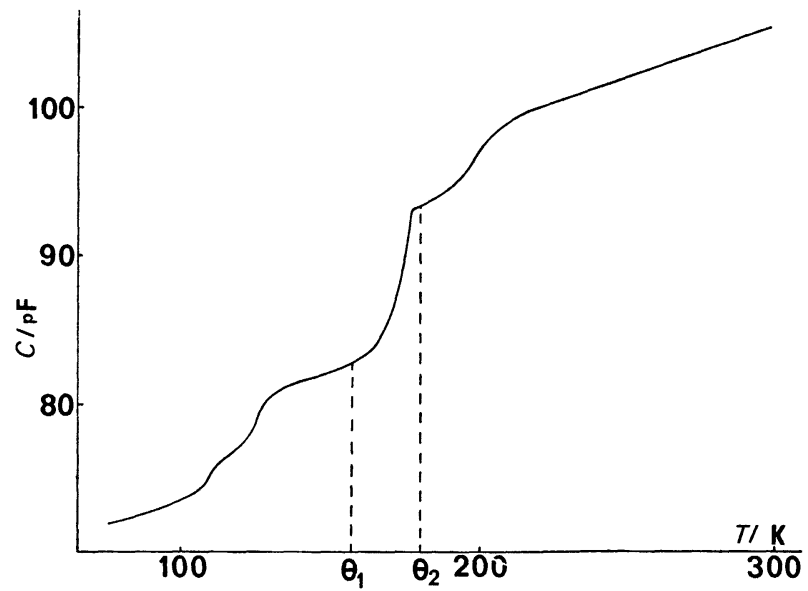

Fig. 3. - Courbe expérimentale de thermocapacitance sur thyristor (structure II). L'accroissement de capacité entre $\theta_{1}$ et $\theta_{2}$ est interprété au paragraphe 2.2.

$\theta_{2}$ dont la différence est voisine de $20 \mathrm{~K}$. La réalisation des transitoires isothermes de capacité pour toute température $T_{\mathrm{e}}<T<\theta_{1}$ n'est en pratique pas gênée par la contribution de la jonction $\mathrm{np}^{+}$dont la capacité, constante au cours du temps, a une valeur $C_{2} \gg C_{1}$, en sorte que $C(t) \simeq C_{1}(t)$ (voir Fig. 4). Par cette technique, on ne peut pas caractériser un centre dont

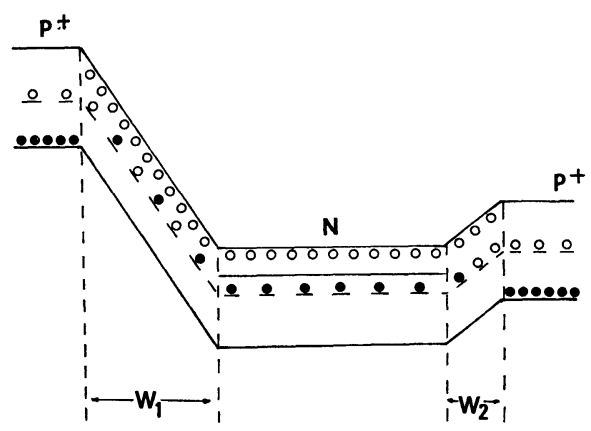

Fig. 4. - Schéma de bande d'une structure $\mathrm{p}^{+} \mathrm{np}^{+}$polarisée.

les niveaux d'énergie se vident de porteurs pour une température $T_{\mathrm{i}}$ telle que $\theta_{1}<T_{\mathrm{i}}<\theta_{2}$. Mais on peut toutefois déterminer s'il existe ou non de tels centres dans la structure ; il suffit pour cela de comparer les densités totales de charges ionisées en $\theta_{1}$ et $\theta_{2}$, accessibles par des mesures $C(V)$ (4). Dans nos échantillons aucun centre similaire n'a été détecté.

La thermocapacitance est donc applicable aux thyristors sauf dans un domaine de température $\left(\theta_{1}, \theta_{2}\right)$ que l'on détermine aisément : en effet cet intervalle se manifeste par un saut de capacité d'allure originale que l'on situe dans tous les cas sans difficulté (Fig. 3).

3. Fabrication des dispositifs. - La figure 5 montre les profils de diffusion de structures correspondant à diverses étapes de la fabrication d'un thyristor. Toutes
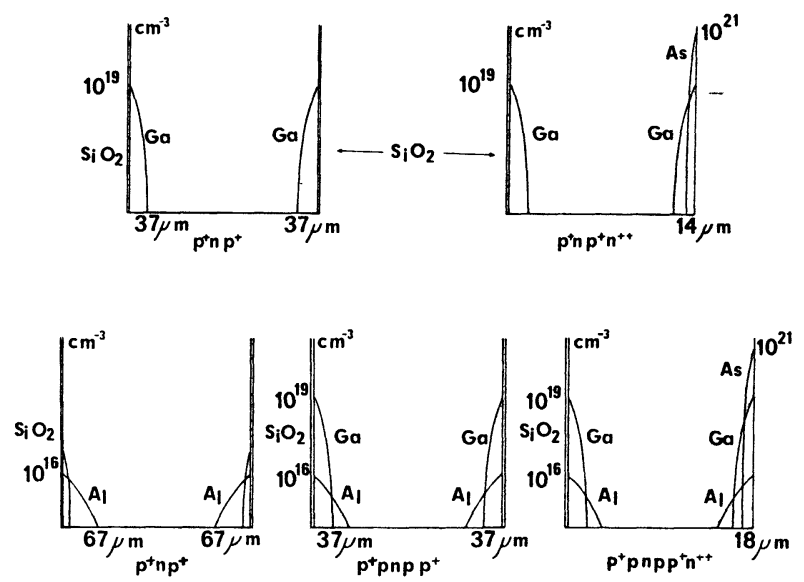

Fig. 5. - Profils de diffusion des cinq types de structures correspondant à diverses étapes de la fabrication d'un thyristor.

ces structures ont été réalisées sur des tranches issues du même lingot de silicium type $N$ de résistivité 40 à $50 \Omega \times \mathrm{cm}$. L'épaisseur des tranches est de $370 \mu \mathrm{m}$ et leur surface a été rodée. La préparation de chaque structure a été conduite de la façon suivante [8]:

Structure I. - Diffusion simultanée en tube scellé, d'arsenic et de gallium à partir du composé intermétallique AsGa $\left(36 \mathrm{~h} / 1180^{\circ} \mathrm{C}\right)$ sur tranche oxydée non ouverte (afin d'éviter la diffusion d'arsenic). On 
obtient donc une structure $\mathrm{p}^{+} \mathrm{np}^{+}$. Les échantillons ont été refroidis à une vitesse de $60^{\circ} \mathrm{C} / \mathrm{h}$.

Structure II. - Diffusion identique à celle indiquée ci-dessus, mais avec tranche ouverte (diffusion localisée d'arsenic). On obtient donc une structure $\mathrm{p}^{+} n \mathrm{p}^{+} \mathrm{n}^{++}$. Les échantillons ont été refroidis à une vitesse de $60^{\circ} \mathrm{C} / \mathrm{h}$.

Structure III. - Diffusion d'aluminium en tube scellé $\left(9 \mathrm{~h}\right.$ à $\left.1250^{\circ} \mathrm{C}\right)$ puis oxydation. On obtient une structure $\mathrm{p}^{+} \mathrm{np}^{+}$. Les échantillons ont été refroidis à une vitesse de $150^{\circ} \mathrm{C} / \mathrm{h}$.

Structure IV. - A partir de structures du type III, on effectue une diffusion simultanée d'arsenic et de gallium $\left(36 \mathrm{~h} / 11^{\circ} \mathrm{C}\right)$ sur tranche non ouverte. On obtient une structure $\mathrm{p}^{+} \mathrm{np}^{+}$. Les échantillons ont été refroidis à une vitesse de $150^{\circ} \mathrm{C} / \mathrm{h}$.

Structure V. - A partir de structures du type III, on effectue une diffusion simultanée d'arsenic et de gallium $\left(36 \mathrm{~h} / 1180^{\circ} \mathrm{C}\right)$ sur tranche ouverte. On obtient une structure $\mathrm{p}^{+} \mathrm{pnpp}^{+} \mathrm{n}^{++}$. Les échantillons ont été refroidis à une vitesse de $60^{\circ} \mathrm{C} / \mathrm{h}$.

Des rondelles de diamètre $\varnothing=10 \mathrm{~mm}$ ont été découpées dans ces structures et plusieurs échantillons de chaque structure ont été analysés par thermocapacitance. Les résultats sont présentés dans le chapitre suivant.

4. Résultats expérimentaux et discussion. - Trois niveaux d'énergie (numérotés de 1 à 3) apparaissent dans tous les dispositifs (sauf pour la structure $I$ où seuls les niveaux 2 et 3 sont présents). Les résultats expérimentaux sur les coefficients d'émission sont présentés sur la figure 6. Les niveaux d'énergie, et les sections de capture calculées à partir de (6) sont présentes dans le tableau I. Les profils de densité des niveaux 2 et 3 sont représentés sur la figure 7, (pour le niveau 1, nous n'avons déterminé que la densité moyenne).

Nos résultats peuvent être comparés à ceux de Yau et Sah [3] qui ont travaillé sur des jonctions $\mathrm{p}^{+} \mathrm{n}$. Les niveaux 2 et 3 sont bien ceux que Yau et Sah associent à la trempe quenched-in centers car :

- l'accord avec Yau et Sah pour les valeurs de

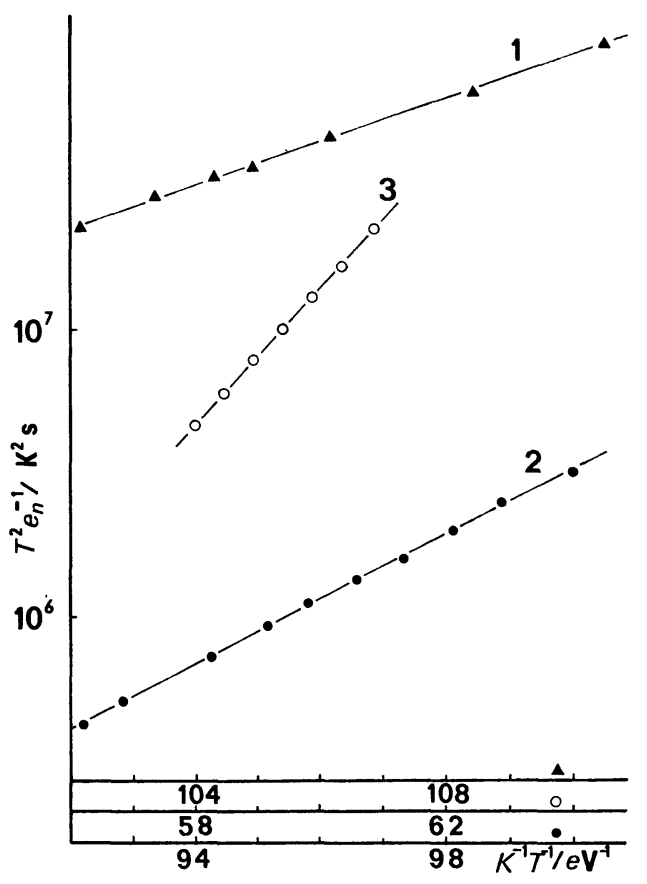

Fig. 6. - Résultats expérimentaux pour les coefficients d'émission des niveaux 1,2 et 3 .

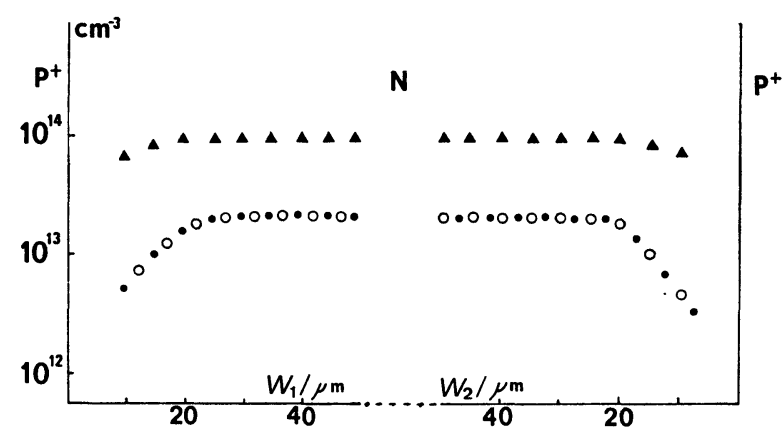

Fig. 7. - $\Delta$ Densité $N_{\mathrm{D}}$ de centres peu profonds ; Densité du centre associé au niveau $2 ; 0$ Densité du centre associé au niveau 3.

$E_{\mathrm{C}}-E_{\mathrm{T}}$ est excellent pour le niveau 3 et bon pour le niveau 2 (voir Tableau I) ;

- les profils de densité pour les niveaux 2 et 3

(Fig. 7) sont identiques, ce qui a aussi été observé par

\section{TABLEAU I}

Les résultats de L. D. Yau et C. T. Sah sont indiqués entre crochets [3].

$\begin{array}{ccc}\text { Centre } & \mathrm{E}_{\mathrm{c}}-\mathrm{E}_{\mathrm{i}}(\mathrm{meV}) & A\left(\mathrm{~s}^{-1}\right)\left({ }^{*}\right) \\ \overline{1} & 160 \pm 5 & 4,9 \times 10^{4} \\ 2 & 257 \pm 4 & 4,1 \times 10^{9} \\ & {[264 \pm 3]} & {\left[5,5 \times 10^{10}\right]} \\ 3 & 542 \pm 2 & 8,7+10^{11} \\ & {[542 \pm 4]} & {\left[15 \times 10^{11}\right]}\end{array}$

Section de capture $\sigma\left(\mathrm{cm}^{2}\right)$

Nature du défaut

$$
\begin{array}{r}
1,6 \times 10^{-22} \\
1,32 \times 10^{-17} \\
2,8 \times 10^{-15}
\end{array}
$$

Défaut associé à la trempe

Défaut associé à la trempe

(*) $A$ est donné par

$$
e_{\mathrm{n}}=A\left(\frac{T}{300}\right)^{2} \exp -\frac{E_{\mathrm{c}}-E_{\mathrm{i}}}{k T} .
$$


Yau et Sah (ceci suggère que ces deux niveaux sont associés au même centre);

- ces niveaux sont présents dans tous les échantillons quels que soient les diffusions ou traitements thermiques subis (voir Tableau II).

\section{TABLEAU II}

Densités de centres dans la zone centrale des dispositifs $\left(\mathrm{cm}^{-3}\right)$
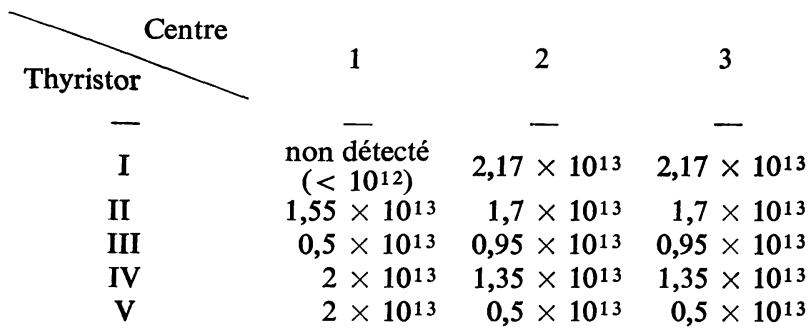

Yau et Sah ont montré que la vitesse de refroidissement a une grande influence sur la densité de ces niveaux. Le fait que les densités varient d'un échantillon à l'autre pour les niveaux 2 et 3 peut avoir son origine dans de petites variations de la vitesse de refroidissement.

Le niveau 1 ne peut être identifié à l'un de ceux trouvés par Sah et Wang [4] qui ont travaillé sur des jonctions p-n et des diodes Schottky. La faible section de capture électronique du centre associé à ce niveau permet de suggérer que ce centre est chargé (-) ou (- -) lorsqu'il est vide d'électron.
Si au cours de la fabrication, des impuretés polluantes produisant des niveaux d'énergie dans la bande interdite (autres que les niveaux 1, 2 et 3 ) sont introduites, la sensibilité de notre méthode permet de conclure que leur densité est $\lesssim 10^{12} \mathrm{~cm}^{-3}$.

5. Conclusion. - Nous avons déterminé dans quelles conditions expérimentales la thermocapacitance et la méthode des transitoires de capacité dans l'obscurité sont applicables aux dispositifs de puissance au silicium. En appliquant ces méthodes à diverses étapes de l'élaboration d'un thyristor, nous trouvons trois niveaux d'énergie dans la bande interdite. Deux de ces niveaux, présents dans tous les échantillons, sont associés à un ou plusieurs défauts liés à la trempe et caractérisés pour la première fois par Yau et Sah. Le niveau 3, qui est proche du milieu de bande, peut avoir une grande contribution au courant de génération inverse. C'est pourquoi nous pensons qu'il est important de développer de telles techniques de contrôle ayant une bonne résolution en énergie (quelques $\mathrm{meV}$ ) et une grande sensibilité (densités $\gtrsim 10^{12} \mathrm{~cm}^{-3}$ ) dans le but de détecter des défauts incontrôlés qui, même en faible densité, peuvent jouer un grand rôle dans le fonctionnement inverse des dispositifs.

Remerciements. - Les auteurs tiennent à remercier MM. Salbreux et Pezzani, Silec-Semiconducteurs, usine de Villejuif, 30, av. de la République, 94800 Villejuif, pour la préparation des échantillons.

\section{Bibliographie}

[1] Pugnet, M., Barbolla, J., Brabant, J. C. et Brousseau, M., article à paraître à Solid-State Electron.

[2] Sah, C. T., Chan, W. W., Fu, H. S. et Walker, J. W., Appl. Phys. Lett. 20 (1972) 193.

[3] YaU, L. D. et SAH, C. T., Solid-State Electron. 17 (1974) 193.

[4] Sah, C. T. et Wang, C. T., J. Appl. Phys. 46 (1975) 1767.
[5] SAH, C. T., Forbes, L., Rosier, L. L. and TASCh, A. F. JR, Solid-State Electron. 13 (1970) 759.

[6] SAH, C. T. et ReDDI, V. G. K., IEEE Trans. Electron Devices, ED-11 (1964) 345.

[7] SaH, C. T. et Fu, H. S., Phys. Stat. Sol. (a) 11 (1972) 297.

[8] Ces structures ont été préparées par MM. Salbreux et Pezzani, Silec-Semiconducteurs, Usine de Villejuif, 30, av. de la République, 94800 Villejuif. 